\title{
Neutron Star Population Dynamics
}

James M. Cordes, David Chernoff and Zaven Arzoumanian

Astronomy Department, Cornell University, Ithaca, NY 14853

\begin{abstract}
We summarize our efforts at understanding the galactic neutron star (NS) population using likelihood and bayesian analyses. These include determination of the velocity distribution of young, high-magnetic field objects; the spatial, velocity, period and luminosity distributions of millisecond pulsars; and a full analysis of high-field objects including modeling of pulsars' radio beams with the implicit assumption that such objects, for fixed period and period derivative, are standard candles.
\end{abstract}

\section{KINEMATICS OF YOUNG PULSARS}

- A likelihood analysis is used that takes into account:

1. proper motion measurements + errors;

2. distance estimates + errors;

3 . information about radial velocities that is contained in $z=D \sin b$;

4. multicomponent velocity models;

5. an odds ratio is used to determine the model that best fits the data with a minimum of parameters.

- A single-gaussian model and a triple-gaussian model are disfavored by the data.

- The best fit model is a two component 3D gaussian with a single birth $z$ scale height:

1. component 1: $\sigma_{V_{1}}=175_{-30}^{+20} \mathrm{~km} \mathrm{~s}^{-1}$.

2. component 2: $\sigma_{V_{2}}=800 \pm 200 \mathrm{~km} \mathrm{~s}^{-1}$.

3. birth scale height: $h_{z}=0.13 \mathrm{kpc}$.

- About $25 \%$ of known pulsars will escape the Galaxy (for escape speed = $500 \mathrm{~km} \mathrm{~s}^{-1}$ ).

- We find no significant correlation between the 3D velocities and any combination of $\mathrm{P}$ and $\dot{\mathrm{P}}$.

\section{KINEMATICS OF MILLISECOND PULSARS}

- A likelihood analysis is done on $8 \mathrm{MSP}$ surveys that takes into account:

1. MSP discoveries and non-detection survey beam areas; 
2. search sensitivity vs $P, \mathrm{DM}, \ell, b, \nu, \mathrm{D}=$ distance;

3. errors on distance estimates;

4. interstellar scintillations, which modulate the intensity and yield a net increase in surveyed volume, by $\sim 30 \%$.

- We consider power-law models for the distributions of MSPs in $P$ and in pseudo luminosity $L_{p}=D S^{2}$.

- We consider a galactic disk-only model (plane parallel) that is exponential in $\approx$ with scale height $\sigma_{z}$.

- We also consider disk+halo models.

- Results include:

1. MSP periods are distributed $\propto P^{-2 \pm 0.3}$.

2. Pseudo luminosities are distributed $\propto L_{p}^{-2 \pm 0.3}$.

3. The scale height is $\sigma_{z}=0.5 \pm 0.15 \mathrm{kpc}$

4. The scale height corresponds to an $\mathrm{rms} z$ velocity $\sigma_{V_{z}} \approx 50 \mathrm{~km} \mathrm{~s}^{-1}$ and a $3 \mathrm{D}$ velocity $\sim 90 \mathrm{~km} \mathrm{~s}^{-1}$.

5. The minimum period is $P_{\min }>0.65 \mathrm{~ms}$ ( $99 \%$ confidence).

6. The space density of MSPs at $\mathrm{z}=0$ is $n_{d} \sim 28_{-11}^{+14} \mathrm{kpc}^{-3}$.

7. In a disk of radius $10 \mathrm{kpc}$, there are $10^{4.0} \mathrm{MSPs}$.

8. The corresponding birth rate (in $10 \mathrm{Gyr}$ ) is $\dot{n} \sim 10^{-6.0} \mathrm{yr}^{-1}$.

9. In a disk + halo model, the likelihood is maximum for a negligible halo population.

\section{MONTE-CARLO MODELING OF THE PULSAR POPULATION}

We have also undertaken a comprehensive study of the NS population that removes all selection effects, including those on the pulsar velocity distribution. We do so by performing a forward analysis whereby we create pulsars, search for them in pseudo surveys that have properties like those actually performed, and compare the detections with the known pulsars. Our scheme consists of: (1) integrating NS orbits in a model for the galactic potential; (2) generating pulsars at a constant birth rate; (3) modeling radio beams with core and conal components; (4) adopting a deterministic relationship between (true) radio luminosity and $P$ and $\dot{P} ;(5)$ assuming random orientation angles between the magnetic moment, spin axis and line of sight; (6) Smearing out the intrinsic pulse shape by dispersion over a channel bandwidth and by scattering; (7) Comparing modeled and actual pulsars with a numerically derived likelihood function.

The key assumption in (4) is that radio pulsars of a given $\mathrm{P}$ and $\dot{P}$ are standard candles. Results of our program will be reported elsewhere. 